\title{
Thymoquinone induces cell proliferation inhibition and apoptosis in acute myeloid leukemia cells: role of apoptosis-related WT1 and BCL2 genes
}

\author{
Mishary G. Musallia, Mohammed A. Hassana,b, Ryan A. Sheikha, Abdulaziz A. Kalantana, \\ Majed A. Halwanic, Mustafa Zeyadia, Salman Hosawia, Mahmoud Alhosina,d* \\ ${ }^{a}$ Biochemistry Department, Faculty of Science, King Abdulaziz University, Jeddah, Saudi Arabia. \\ ${ }^{b}$ Department of Basic Medical Sciences, College of Medicine and Health Sciences, Hadhramout University, Mukalla, Yemen. \\ ${ }^{c}$ Nanomedicine Department, King Abdullah International Medical Research Center (KAIMRC), King Saud bin Abdulaziz University for \\ Health Sciences. \\ ${ }^{d}$ Cancer Metabolism and Epigenetic Unit, Faculty of Science, King Abdulaziz University, Jeddah, Saudi Arabia.
}

Received: 03 April 2019 / Revised: 17 May 2019 / Accepted: 02 June 2019

\begin{abstract}
Acute myeloid leukemia (AML) is an aggressive and heterogeneous disease characterized by an abnormal proliferation and impaired differentiation of the myeloid precursor cells. The outcome for most AML patients remains poor with high relapse rates and chemotherapy remains the first line treatment for AML. The Wilms tumor wt1 and the anti-apoptotic BCL2 genes are upregulated in AML and are known to be involved in apoptosis inhibition. In the present study we evaluated the molecular mechanisms underlie the anti-proliferative and proapoptotic activities exerted by thymoquinone (TQ), the major biologically active compound of the black seed oil on acute myeloid leukemia (AML) cell line-HL60. Cell proliferation was determined by WST-1 assay and apoptosis rate was assessed by flow cytometry using annexin-V/7AAD staining. The expression of target genes was analyzed by real-time RT-PCR analysis. TQ significantly reduced HL60 cell viability and induced apoptosis in a dose and time-dependent manner. In order to decipher the molecular mechanisms underlie the anti-cancer activities induced by TQ in AML cells, we investigated its effect on the expression of WT1 and BCL2 genes. TQ significantly decreased the expression of WT1 and BCL2 genes in a dose and time-dependent manner. In summary, these findings suggest that $\mathrm{TQ}$ induces cell proliferation inhibition and apoptosis in acute myeloid leukemia cells most likely through targeting the apoptosis-related $W T 1$ and BCL2 genes and also suggest that TQ could be a promising strategy for AML therapy.
\end{abstract}

\section{Keywords:}

Thymoquinone, Apoptosis, BCL2, WT1, HL-60, AML.

(C) Euraass 2019. All rights reserved.

\section{Introduction}

Acute myeloid leukemia (AML) is an aggressive and heterogeneous disease characterized by an abnormal proliferation and impaired differentiation of the myeloid precursor cells [1]. The outcome for most AML patients remains poor with high relapse

* Corresponding author. E-mail address: malhaseen@kau.edu.sa (M. Alhosin); Ph: +966 597959354

Available online: 30 June 2019

DOI: https://doi.org/10.34154/2019-EJCS-0101-02-09/euraass

Cite this: Eur. J. Cell Sci. 2019, 1(1), 02 - 09.

ISSN-E: 2679-3350.

(C) European Academy of Applied and Social Sciences. Euraass - 2019. All rights reserved. 
rates and chemotherapy remains the first line treatment for AML [1, 2]. The Wilms tumor WT1 and the anti-apoptotic BCL2 genes are upregulated in patients with most forms of acute leukemia including AML and are known to be involved in the inhibition of apoptosis [24]. WT1 was shown to positively regulate the expression of BCL2 leading to failure in apoptosis as well as resistant to chemotherapyinduced apoptosis [5, 6]. In this context, the upregulation of WT1 was found to inhibit p53-mediated apoptosis in Saos-2 cells, an osteosarcoma cell line through a direct interaction with p53 [7]. Considering the fact that the defect in apoptosis leads lo malignant transformation, the overexpression of WT1 could potentiate carcinogenesis by inhibiting the apoptosis. In agreement with this hypothesis, high expression levels of WT1 was found in several solid tumors [8-10] and in leukemia including AML [5, 8, 11, 12]. WT1 was detected in the bone marrow of $70-80 \%$ of AML patients but not in bone marrow of healthy controls [13, 14] and also suggested as a possible marker for the detection of leukemic blast cells [15]. WT1 can inhibit apoptosis in cancer cells through several mechanisms including targeting the anti-apoptotic BCL2 $[5,16,17]$. Like WT1, high expression levels of BCL2 were also detected in $\mathrm{AML}$ and considered as prognostic factor in this type of leukemia [18-20]. Considering the fact that the upregulation of BCL2 observed in AML is related to the overexpression of WT1, finding new types of inhibitors of WT1/BCL2 pathway could be a promising strategy for AML therapy.

Many purified components extracted from medicinal plants have shown beneficial therapeutic potentials in the recent years and widely used as an alternative to chemical drugs. Thymoquinone (TQ), the major bioactive component of the essential oil of black seeds have shown promising inhibitory effects on a large number of solid and leukemia tumors through targeting several pathways such as those involved in cell cycle, apoptosis, ROS generation, metastasis and angiogenesis [21-26].

Consequently, the aim of the present study was to evaluate the molecular mechanisms underlie the anti-proliferative and proapoptotic activities exerted by TQ on acute myeloid leukemia (AML) cell line-HL60. Our results showed that TQ reduced HL60 cell viability and induced apoptosis in a time and dose-dependent manner. TQ-induced apoptosis in HL60 was associated with a decrease in the expression of $W T 1$ and BCL2 genes. These findings indicate that $\mathrm{TQ}$ induces cell proliferation inhibition and apoptosis in acute myeloid leukemia cells most likely through targeting the apoptosis-related WT1 and BCL2 genes and also suggest that TQ could be a promising strategy for AML therapy.

\section{Materials \& Methods}

\subsection{Cell culture and treatment}

Acute myeloid leukemia (AML) cell line HL60 were purchased from American Type Culture Collection (ATCC, Manassas, VA, USA). Cells were maintained in RPMI1640 (UFC-Biotech, Riyadh,
SA) supplemented with 15\% (v/v) FBS, penicillin (100 IU/ml) and streptomycin $(100 \mu \mathrm{g} / \mathrm{ml})$. Cells were maintained in a humidified incubator containing $5 \% \mathrm{CO}_{2}$ at $37^{\circ} \mathrm{C}$. For all treatments, a $10 \mathrm{mM}$ solution of TQ (Sigma-Aldrich, Louis, MO, USA) was prepared in 10\% DMSO (DiMethylSulfOxide; Millipore, Molsheim, France) and appropriate working concentrations were prepared with cell culture medium. The final concentration of DMSO was always less than $0.1 \%$ in both control and treated conditions.

\subsection{Cell proliferation assay}

The effect of $\mathrm{TQ}$ on cell proliferation was analyzed by a colorimetric cell proliferation assay using WST-1 Cell Proliferation Reagent Kit (Sigma-Aldrich, USA). Briefly, the cells were seeded in 96-multiwell plates at a density of $4 \times 10^{4} /$ well. After $24 \mathrm{~h}$ of incubation, the cells were exposed to different concentrations of TQ for the desired time periods. Cell proliferation rate then was evaluated through a rapid WST-1 reagent. After incubation for the desired time periods, $10 \mu \mathrm{L}$ of the WST-1 solution was added and incubated for an additional $3 \mathrm{~h}$ at $37^{\circ} \mathrm{C}$. Finally, the absorbance was read at $450 \mathrm{~nm}$ with a microplate ELISA reader (ELX800 ${ }^{\mathrm{TM}}$ Biotek, USA) and the results were analyzed by the Gen5 software (Biotek, USA). The reaction based on the cleavage of the tetrazolium salt WST-1 to formazan by cellular mitochondrial dehydrogenases. The quantity of formazan dye in the medium is directly proportional to the number of viable metabolically active cells. The percentage of cell viability was calculated by assuming control (untreated) samples as $100 \%$ viable.

\subsection{Apoptosis Assay}

To study the apoptosis, HL60 cells were seeded in 96-well plates at a density of $4 \times 10^{4}$ cells/well, grown for $24 \mathrm{~h}$ and exposed to different concentrations of TQ for the desired time periods. Cell apoptosis rate was assessed using the Annexin V Binding Guava Nexin ${ }^{\circledR}$ Assay by capillary cytometry (Guava Easycyte Plus HP system, with absolute cell count and six parameters) following the manufacturer's recommendations (Guava Technologies Inc, Hayward, CA, USA). Guava Nexin® Assay utilizes Annexin V-PE.

\subsection{Real-time RT-PCR analysis}

Real-time RT-PCR analysis was described elsewhere [27]. Briefly, the cells were treated with different concentrations of TQ for the desired time periods. Then, total RNAs were purified and subjected to reverse transcription using Oligo (dt) (Sigma) and Superscript II reverse transcriptase (Invitrogen). Quantitative realtime PCR was done with the LightCycler 480 SYBR Green I Master kit (Roche Diagnostics) and the Mastercycler Realplex apparatus (Eppendorf, Montesson, France). The results were normalized with RPL11 mRNA. The sequences of the primers for PCR amplification were: WT1 (sense: 5 ' -GCTGTCCCACTTACAGATGCA-3 ' ; antisense: $5^{\prime}$-TCAAAGCGCCAGCTGGAGTTT-3 ' ); BCL2 (sense: 5' -TCCGCATCAGGAAGGCTAGA-3' , antisense: 5' AGGACCAGGCCTCCAAGCT-3 ' ), RPL11 (sense: 5 ' AGCCAAGGTCTTGGAGCAGCTTA-3 ', antisense: 5 ' - 
TTGGGCCTCTGACAGTACAGTGAACA-3 ' ). Amplicons were size controlled on agarose gel and purity was assessed by analysis of the melting curves at the end of the RT-PCR reaction.

\subsection{Statistical Analysis}

All the data were presented as Mean SEM of triplicates done in the same experiment or an average of at least three separate experiments. The differences between the control and the treated were analyzed by Student's t-test (two-tailed) using GraphPad Prism 6 (Graph Pad Software, SanDiego, USA) and the significant differences were indicated as ${ }^{*} p<0.05,{ }^{* *} p<0.01,{ }^{* *} p<0.001$, ${ }^{* * * *} p<0.0001$.

\section{Results}

\subsection{TQ reduced $H L 60$ cell viability through dose and time- dependent mechanism}

We first studied the anti-proliferative effect of TQ in HL60 cells. For this purpose, HL60 cells were incubated with increasing concentrations of $T Q$ for $24 \mathrm{~h}$ (Fig. 1A). We found that TQ began to significantly inhibit cell proliferation from $10 \mu \mathrm{M}$ (Fig. 1A). At a higher concentration $30 \mu \mathrm{M}, \mathrm{TQ}$ significantly decreased HL60 viability by about $60 \%$ (Fig. $1 \mathrm{~A}$ ). Then, a kinetic analysis of $\mathrm{TQ}$ on cell proliferation in HL60 cells was performed. For this objective, cells were exposed to $30 \mu \mathrm{M}$ of TQ (Fig. 1B). TQ significantly decreased cell proliferation of HL60 starting from $1 \mathrm{~h}$ (Fig. 1B). At 6 $\mathrm{h}$, the reduction in cell viability reached approximately $25 \%$ (Fig. $1 B)$. These findings indicate that $T Q$ induces a dose and time- dependent inhibition of HL60 cell proliferation.

\subsection{TQ induced dose and time-dependent apoptosis in HL60 cells}

We then investigated the apoptotic effect of TQ in HL60 cells under the same experimental conditions. Firstly, cells were exposed to increasing concentrations of TQ for $24 \mathrm{~h}$ (Fig 2). We observed that $\mathrm{TQ}$ began to significantly induce early and late apoptosis at $5 \mu \mathrm{M}$, (Fig. 2). At $30 \mu \mathrm{M}, 65 \%$ of HL60 cells were in early apoptosis stage (Fig. 2A, B) and $21 \%$ in late apoptosis (Fig. $2 \mathrm{~A}, \mathrm{C})$.

Events in each of the four quadrants were: Lower-left quadrant: viable cells, not undergoing detectable apoptosis (Annexin V-PE negative and 7-AAD negative cells). Lower-right quadrant: cells in the early stage of apoptosis (Annexin V-PE positive and 7-AAD negative cells). Upper right quadrant: cells in the late apoptotic or dead (Annexin V-PE positive and 7-AAD positive cells, their number, expressed as percentage relative to the total cell number, is indicated on the right). Upper-left quadrant: mostly nuclear debris (Annexin VPE negative and 7-AAD positive). The number of apoptotic cells in early stage (B) and late apoptosis and dead cells (C) is expressed as percent relative to the total cell number. Values are shown as means \pm S.E.M. $(n=3) ;{ }^{*} p<0.05,{ }^{* *} p<0.01$, ${ }^{* * *} p<$ 0.001 , versus respective control.

B)

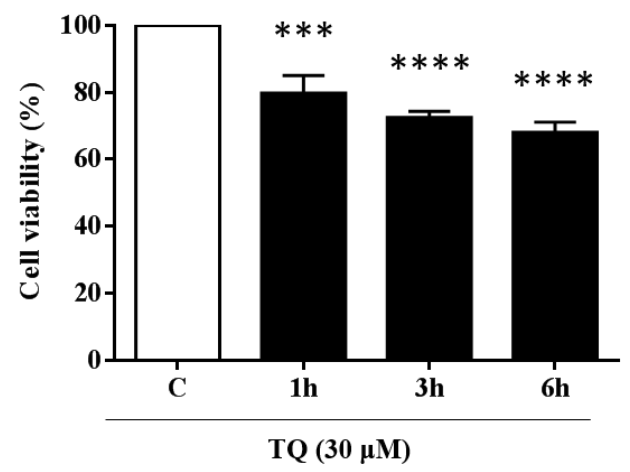

Figure 1: Dose and time-dependent effects of TQ on HL-60 cell viability. Cells were exposed to increasing concentrations of TQ for $24 \mathrm{~h}$ (A) or to $30 \mu \mathrm{M}$ for the indicated times (B). Cell viability rate was assessed by WST-1 assay. The data are representative of three different experiments. Values are shown as means \pm S.E.M. $(n=3) ;{ }^{*} p<0.05,{ }^{* * *} p<0.001,{ }^{* * * *} p<0.0001$ versus respective control. 
A)

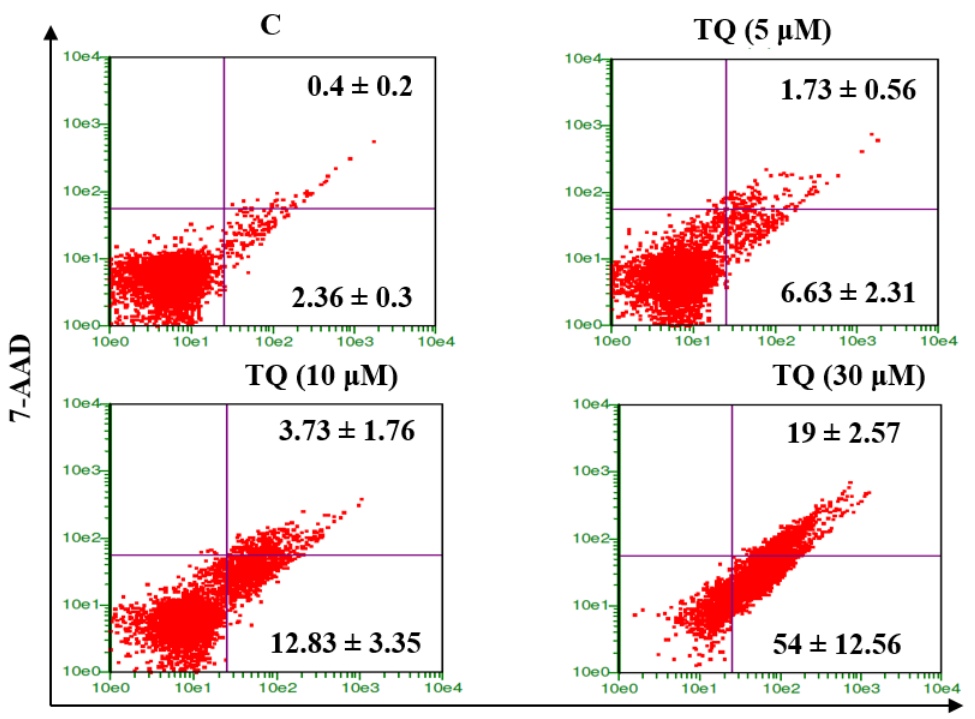

B)

Annexin V-PE

\section{C)}
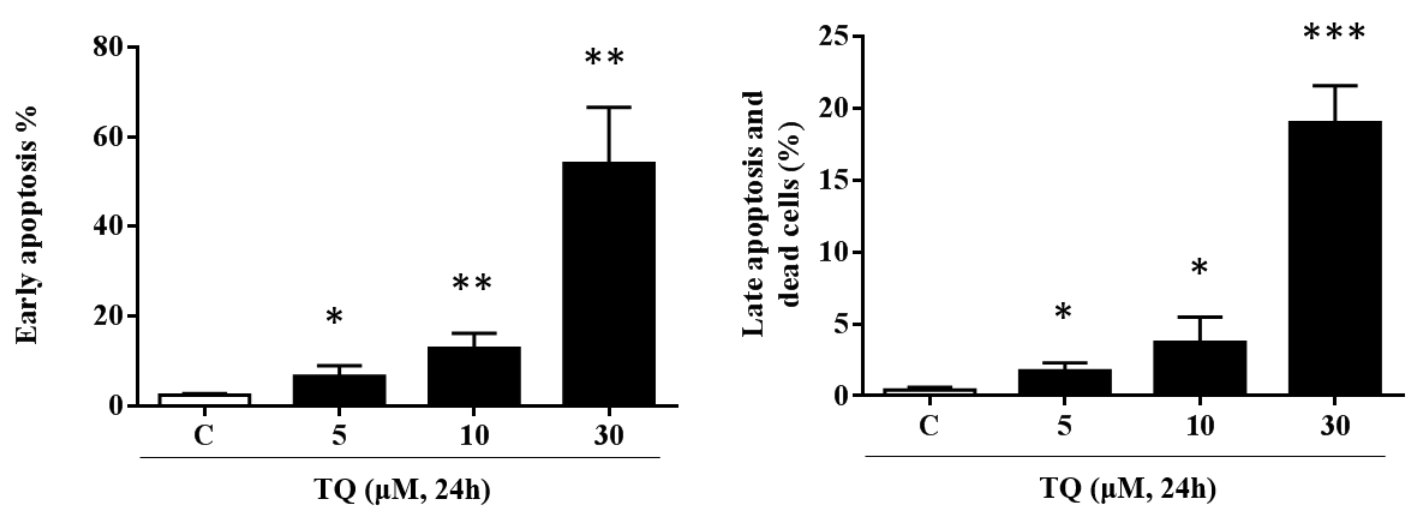

Figure 2: Dose effect of TQ on apoptosis in HL-60 cells. Cells were exposed to increasing concentrations of TQ for $24 \mathrm{~h}$. Apoptosis in HL-60 cells was assessed by flow cytometry using the annexin V-Phycoerythrin (PE) and 7-Amino-Actinomycin (7AAD) staining assay. (A)

Then, we performed a kinetic analysis of TQ on apoptosis in HL60 cells using $30 \mu \mathrm{M}$ of TQ (Fig. 3). Interestingly, TQ significantly induced apoptosis in HL60 starting from $1 \mathrm{~h}$ (Fig. 3A, B). The percentage of early apoptotic cells was approximately $11 \%$ at $1 \mathrm{~h}$ and reached $35 \%$ at $6 \mathrm{~h}$ (Fig. 3A, C). Altogether, these results indicate that TQ-induced HL60 cell proliferation inhibition results in large part from an apoptosis process. 
A)

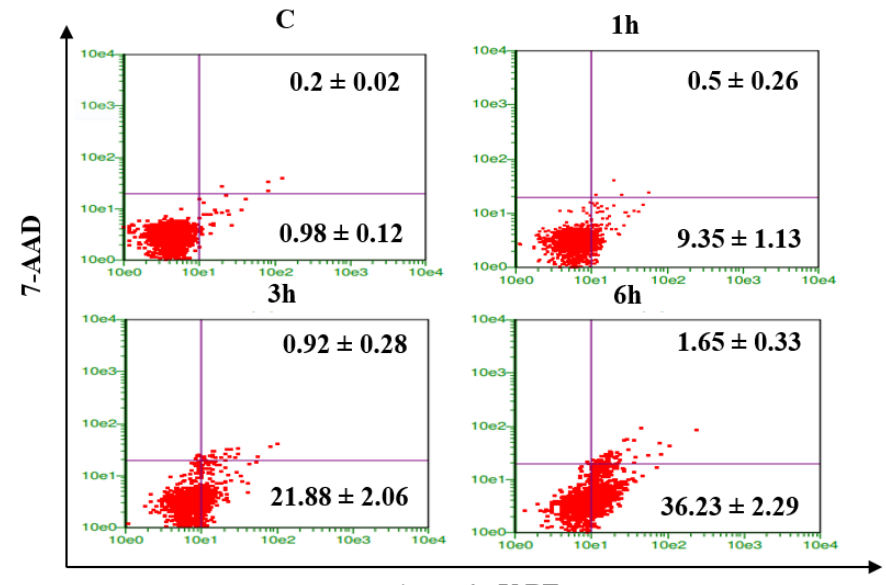

Annexin V-PE

B)

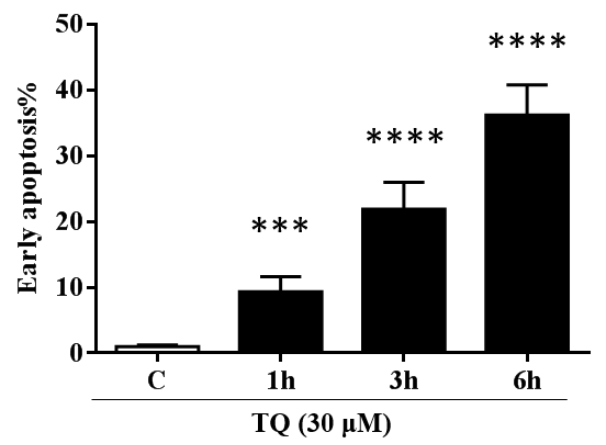

C)

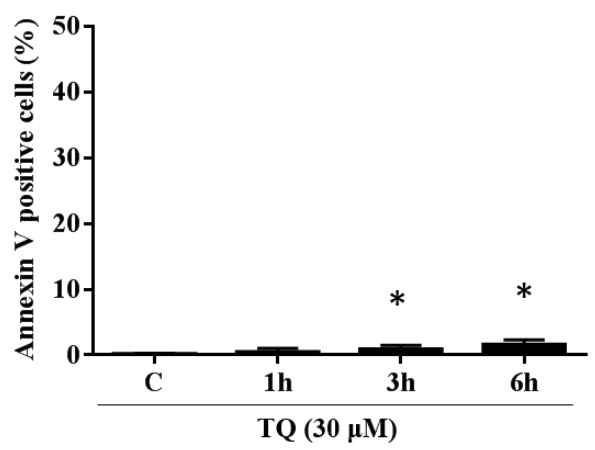

Figure 3: Time-course of the effects of TQ on apoptosis in HL-60 cells. Cells were exposed to $30 \mu M$ of TQ for the indicated times. Apoptosis in HL-60 cells was assessed by flow cytometry using the annexin V-Phycoerythrin (PE) and 7-Amino-Actinomycin (7AAD) staining assay as described in Fig 2 legend. (A) Events in each of the four quadrants. The number of apoptotic cells in early stage (B) and late apoptosis and dead cells $(\mathrm{C})$ is expressed as percent relative to the total cell number. Values are shown as means \pm S.E.M. $(\mathrm{n}=3) ;{ }^{*} p<0.05,{ }^{* \star *} p<0.001,{ }^{* * *} p<$ 0.0001 versus respective control.

\subsection{TQ decreased the expression of WT1 and BCL2 genes in a dose and time-dependent manner.}

As the Wilms tumor WT1 and the anti-apoptotic BCL2 genes are upregulated in $\mathrm{AML}$ and are known to be involved in the inhibition of apoptosis [3, 4], we studied the effect of 5 and $10 \mu \mathrm{M}$ TQ for $24 \mathrm{~h}$ on mRNA expression of WT1 (Fig. 4A) and BCL2 (Fig. 4B) in HL60 using RT-qPCR. We found that mRNA expression of both target genes WT1 (Fig. 4A) and BCL2 (Fig. 4B) was significantly decreased in a dose-dependent manner in HL60 treated with TQ compared with control. Under the same conditions, this effect was associated with a significant increase in apoptosis in
HL60 (Fig. 2) suggesting a significant role for these genes in the pro-apoptotic effects of $\mathrm{TQ}$ in $\mathrm{AML}$ cells. Interestingly, a kinetic analysis of TQ on mRNA expression of WT1 and BCL2 in HL60 cells using $30 \mu \mathrm{M}$ of TQ showed that the expression of WT1 was significantly decreased at $1 \mathrm{~h}$ (Fig. 4C) while the decrease in the expression of BCL2 started to be significant at 3h (Fig. 4D) suggesting that the decrease in the expression of WT1 is an upstream event in the TQ-induced pathway signaling in HL60 cells leading to the downregulation of the expression of BCL2 with subsequent apoptosis. 
A)

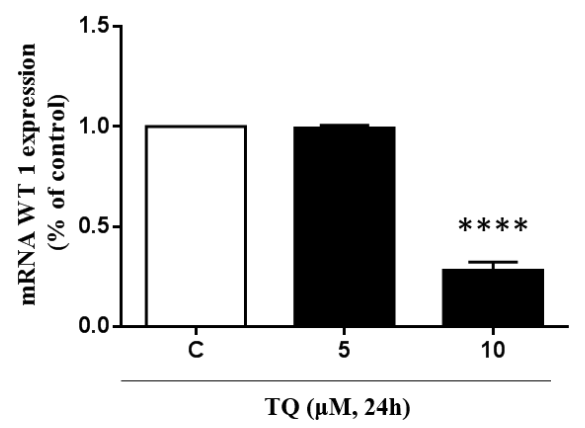

C)

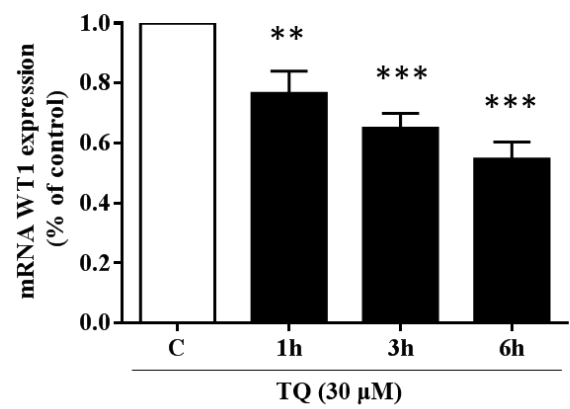

B)

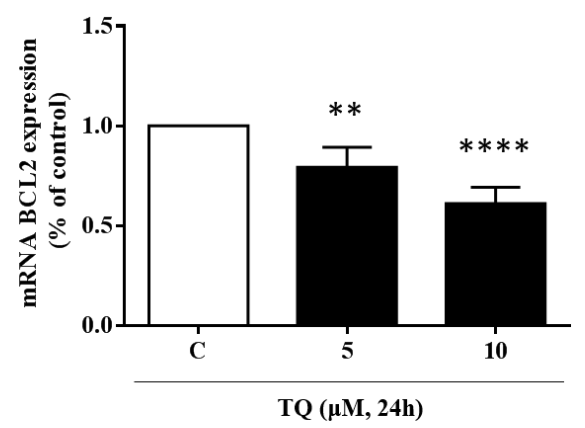

D)

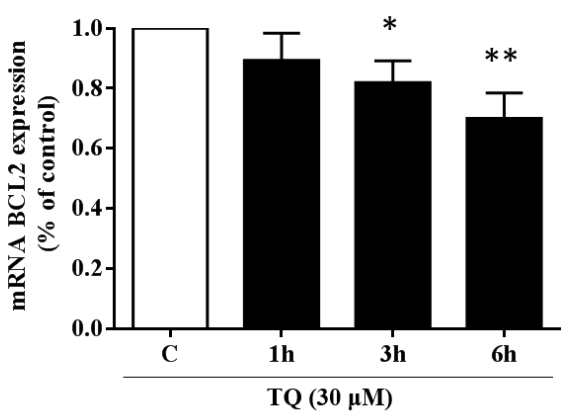

Figure 4: Dose and time-dependent effects of TQ on the expression of WT1 and BCL2 mRNA levels in HL-60 cells. Cells were exposed to increasing concentrations of TQ for $24 \mathrm{~h}(\mathrm{~A}, \mathrm{~B})$ or to $30 \mu \mathrm{M}$ for the indicated times $(\mathrm{C}, \mathrm{D})$. The histograms show the quantification data of mRNA expressions of WT1 $(A, C)$ and BCL2 (B, D), as assessed by real-time PCR. Results are means of three separate experiments performed in triplicate. Values are shown as means \pm S.E.M. $(\mathrm{n}=3) ;{ }^{*} p<0.05,{ }^{* *} p<0.01,{ }^{* * *} p<0.001,{ }^{* * * *} p<0.0001$ versus respective control.

\section{Discussion}

The coordinated overexpression of WT1 and BCL2 genes in cancers and their fundamental role in the inhibition of apoptosis in AML compel us to find new inhibitors of this pathway. Interestingly, the downregulation of WT1 in response to several inhibitory factors such as antisense oligomers is followed by BCL2 downregulation with subsequent induction of apoptosis. Therefore, it is of high interest to find new types of inhibitors of WT1/BCL2 pathway as new strategies of apoptosis induction and thus of anti-cancer therapies. In the present study we evaluated the molecular mechanisms underlie the anti-proliferative and pro-apoptotic activities exerted by TQ on AML cell line-HL60. We found that TQ reduced HL60 cell viability and induced apoptosis in a time and dose-dependent manner. TQ-induced apoptosis was associated with a significant decrease in the expression of WT1 and BCL2

genes in HL60. To our knowledge, it is the first time that WT1/BCL2 pathway is shown as a target of the natural compound, thymoquinone.

High BCL-2 expression was observed in the blasts from AML patients and was correlated with a poor clinical response to chemotherapy in leukemia cells [28]. The knockdown of BCL2 in the blasts of AML patients significantly decreased the expression of the BCL2 protein in $40 \%$ of cases and this effect was associated with an increase in apoptosis rate [28]. Interestingly, the susceptibility of the blasts to apoptosis induced by Cytarabine (Ara$\mathrm{C})$, the most active drug for the treatment of $A M L$ was increased when BCL2 downregulated [28]. Our results showed that TQinduced apoptosis in HL60 was accompanied with a significant decrease in the expression of BCL2 indicating that TQ via inhibiting $B C L-2$ could have clinical potential to increase the chemosensitivity of treatment-resistant AML patients.

Like BCL2, the overexpression WT1 has been shown to have an important role in blast cell survival as well as resistance to chemotherapy in AML [2, 29]. Treating AML cell lines HL60 and K562 with two arsenic compounds arsenic trioxide (As2O3) and 


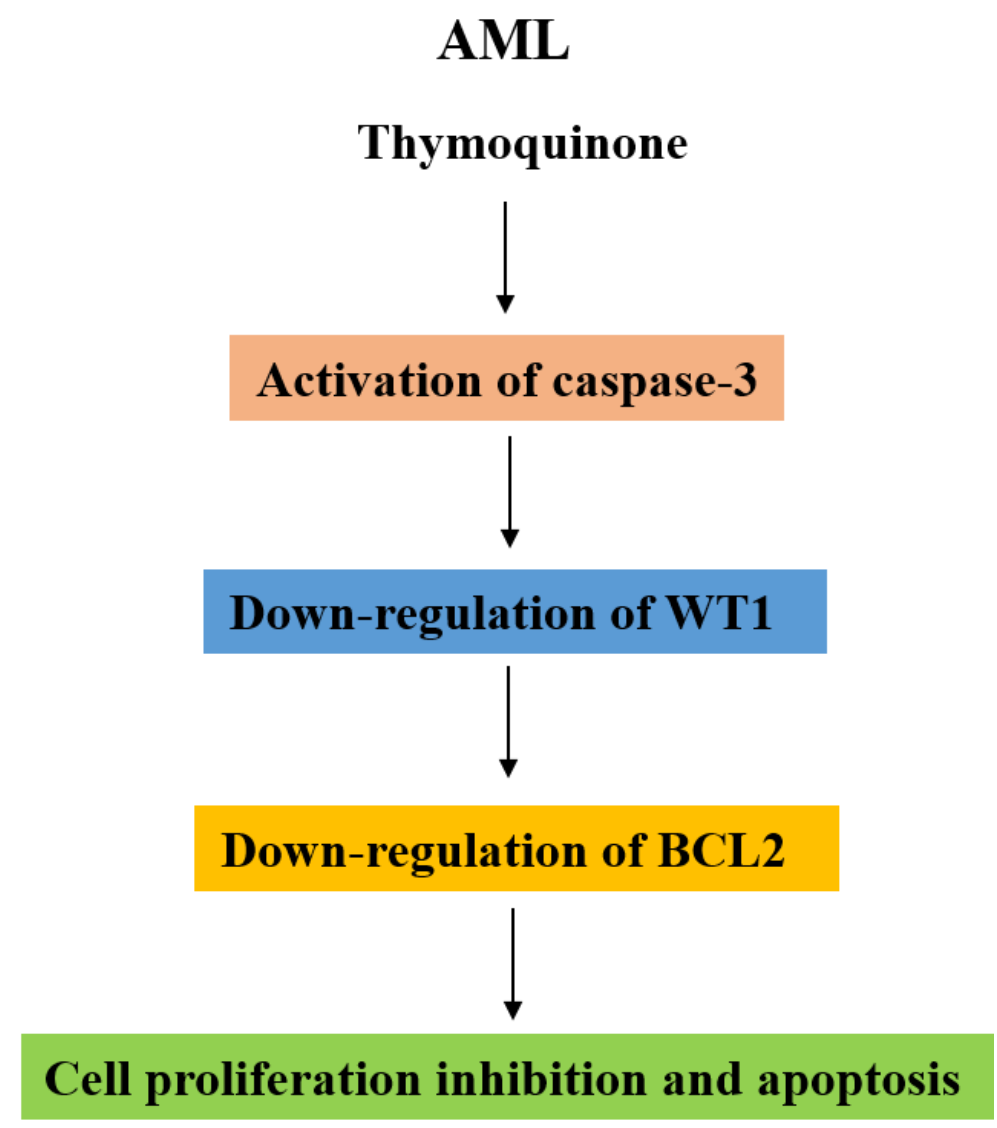

Figure 5: Schematic representation of $\mathrm{WT} 1 / \mathrm{BCl} 2$ pathway regulated by thymoquinone leading to apoptosis in AML. Schematic representation of thymoquinone-induced apoptosis involving WT1 and BCL2 deregulation in acute myeloid leukemia (AML) cells. Thymoquinone induces caspase-3 activation. The activation of the caspase-3 leads to the degradation of WT1 inducing BCL2 downregulation with subsequent cell proliferation inhibition and apoptosis.

sodium arsenite ( $\mathrm{NaAsO} 2)$ induced apoptosis and this effect was accompanied by a decrease in the expression of WT1 at mRNA and protein levels [30]. In the present study we found that TQ induces the downregulation of WT1 in HL60 with subsequent apoptosis indicating that the downregulation of WT1 is a main event in the induction of apoptosis in leukemia cells in response to natural products. In accordance with this hypothesis, the anticancer agent etoposide was shown to induce apoptosis in leukemia cells through the degradation of WT1 and the downregulation of BCL2 protein [5]. Etoposide-induced apoptosis involved the activation of caspase 3 and a decrease in the binding of WT1 to BCL2 promoter [5] and this effect was counteracted by caspase-3 inhibitor. Our previous study showed that TQ induces apoptosis in acute lymphoblastic leukemia cells through the activation of caspase 3 suggesting that the TQ-induced downregulation of WT1 in HL60 in the present study could also result from the activation of caspase 3 which leads to the downregulation of BCL2 and the consequence is apoptosis.

\section{Conclusions}

The present study suggests that the inhibitory effects of TQ on acute myeloid leukemia (AML) involve the downregulation of WT1 through the activation of caspase- 3 leading to the downregulation of BCL2 with subsequent apoptosis (Fig. 5). These results also suggest that $\mathrm{TQ}$ could be used as an inhibitor of WT1/BCL2 pathway which could be a promising strategy for AML as well as other tumors overexpression WT1 and BCL2.

\section{Declaration of Conflicting Interests}

The authors declare that they have no financial conflict of interest. 


\section{Author Contributions}

MA and SH designed the project and wrote the paper. MGM, MAH, RAS and AAK performed research and analyzed data. MAH and MZ MAZ helped with experimental design, data interpretation, and drafting of the paper.

\section{References}

1. Estey E, Dohner H: Acute myeloid leukaemia. Lancet (London, England) 2006, 368(9550):1894-1907.

2. Karakas T, Miething CC, Maurer U, Weidmann E, Ackermann H, Hoelzer D, Bergmann L: The coexpression of the apoptosis-related genes bcl-2 and wt 1 in predicting survival in adult acute myeloid leukemia. Leukemia 2002, 16(5):846-854.

3. Tallman MS, Gilliland DG, Rowe JM: Drug therapy for acute myeloid leukemia. Blood 2005, 106(4):1154-1163.

4. Verhaak RG, Valk PJ: Genes predictive of outcome and novel molecular classification schemes in adult acute myeloid leukemia. Cancer treatment and research 2010, 145:67-83.

5. Ruan J, Gao S, Yang J, Li H, Huang H, Zheng X: WT1 protein is cleaved by caspase-3 in apoptotic leukemic cells. Leukemia \& lymphoma 2018, 59(1):162-170.

6. Mayo MW, Wang CY, Drouin SS, Madrid LV, Marshall AF, Reed JC, Weissman BE, Baldwin AS: WT1 modulates apoptosis by transcriptionally upregulating the bcl-2 proto-oncogene. The EMBO journal 1999, 18(14):3990-4003.

7. Maheswaran S, Englert C, Bennett P, Heinrich G, Haber DA: The WT1 gene product stabilizes p53 and inhibits p53-mediated apoptosis. Genes \& development 1995, 9(17):2143-2156.

8. Bielinska E, Matiakowska K, Haus O: Heterogeneity of human WT1 gene. Postepy higieny i medycyny doswiadczalnej (Online) 2017, 71(0):595-601.

9. Bologna-Molina R, Takeda $Y$, Kuga T, Chosa N, Kitagawa M, Takata T, Ishisaki A, Mikami T: Expression of Wilms' tumor 1 (WT1) in ameloblastomas. Journal of oral science 2016, 58(3):407-413.

10. Eguchi T, Kadota K, Mayor M, Zauderer MG, Rimner A, Rusch VW, Travis WD, Sadelain M, Adusumilli PS: Cancer antigen profiling for malignant pleural mesothelioma immunotherapy: expression and coexpression of mesothelin, cancer antigen 125, and Wilms tumor 1. Oncotarget 2017, 8(44):77872-77882.

11. Mashima K, Oh I, Ikeda T, Toda $Y$, Ito $S$, Umino K, Minakata D, Nakano $\mathrm{H}$, Morita K, Yamasaki $\mathrm{R}$ et al: Role of Sequential Monitoring of WT1 Gene Expression in Patients With Acute Myeloid Leukemia for the Early Detection of Leukemia Relapse. Clinical lymphoma, myeloma \& leukemia 2018, 18(12):e521-e527.

12. Li H, Xing C, Zhou B, Ye H, Feng J, Wu J, Gao S: A regulatory circuitry between miR-193a/miR-600 and WT1 enhances leukemogenesis in acute myeloid leukemia. Experimental hematology 2018, 61:59-68 e55.

13. Brieger J, Weidmann E, Maurer U, Hoelzer D, Mitrou PS, Bergmann L: The Wilms' tumor gene is frequently expressed in acute myeloblastic leukemias and may provide a marker for residual blast cells detectable by PCR. Annals of oncology : official journal of the European Society for Medical Oncology 1995, 6(8):811-816.

14. Miwa H, Beran M, Saunders GF: Expression of the Wilms' tumor gene (WT1) in human leukemias. Leukemia 1992, 6(5):405-409.

15. Brieger J, Weidmann E, Fenchel K, Mitrou PS, Hoelzer D, Bergmann L: The expression of the Wilms' tumor gene in acute myelocytic leukemias as a possible marker for leukemic blast cells. Leukemia 1994, 8(12):2138-2143.
16. Cheema SK, Mishra SK, Rangnekar VM, Tari AM, Kumar R, LopezBerestein G: Par-4 transcriptionally regulates Bcl-2 through a WT1binding site on the bcl-2 promoter. The Journal of biological chemistry 2003, 278(22):19995-20005.

17. Xiong YX, Chen AC, Yao PF, Zeng DY, Lu YJ, Tan JH, Huang ZS, Ou TM: Blocking the binding of WT1 to bcl-2 promoter by Gquadruplex ligand SYUIQ-FM05. Biochemistry and biophysics reports 2016, 5:346-352.

18. Klampfer L, Zhang J, Zelenetz AO, Uchida H, Nimer SD: The AML1/ETO fusion protein activates transcription of BCL-2. Proceedings of the National Academy of Sciences of the United States of America 1996, 93(24):14059-14064.

19. Maung ZT, MacLean FR, Reid MM, Pearson AD, Proctor SJ, Hamilton PJ, Hall AG: The relationship between bcl-2 expression and response to chemotherapy in acute leukaemia. British journal of haematology 1994, 88(1):105-109.

20. Bensi L, Longo R, Vecchi A, Messora C, Garagnani L, Bernardi S, Tamassia MG, Sacchi S: Bcl-2 oncoprotein expression in acute myeloid leukemia. Haematologica 1995, 80(2):98-102.

21. Alhosin M, Abusnina A, Achour M, Sharif T, Muller C, Peluso J, Chataigneau T, Lugnier C, Schini-Kerth VB, Bronner C et al: Induction of apoptosis by thymoquinone in lymphoblastic leukemia Jurkat cells is mediated by a p73-dependent pathway which targets the epigenetic integrator UHRF1. Biochemical pharmacology 2010 , 79(9):1251-1260.

22. Alhosin M, Ibrahim A, Boukhari A, Sharif T, Gies JP, Auger C, Schini-Kerth VB: Anti-neoplastic agent thymoquinone induces degradation of alpha and beta tubulin proteins in human cancer cells without affecting their level in normal human fibroblasts. Investigational new drugs 2012, 30(5):1813-1819.

23. Ibrahim A, Alhosin M, Papin C, Ouararhni K, Omran Z, Zamzami MA, Al-Malki AL, Choudhry H, Mely Y, Hamiche A et al: Thymoquinone challenges UHRF1 to commit auto-ubiquitination: a key event for apoptosis induction in cancer cells. Oncotarget 2018, 9(47):28599-28611.

24. Qadi SA, Hassan MA, Sheikh RA, Baothman OAS, Zamzami MA, Choudhry H, Al-Malki AL, Albukhari A, Alhosin M: ThymoquinoneInduced Reactivation of Tumor Suppressor Genes in Cancer Cells Involves Epigenetic Mechanisms. Epigenetics insights 2019, 12:2516865719839011.

25. Majdalawieh AF, Fayyad MW, Nasrallah GK: Anti-cancer properties and mechanisms of action of thymoquinone, the major active ingredient of Nigella sativa. Critical reviews in food science and nutrition 2017, 57(18):3911-3928.

26. Goyal SN, Prajapati CP, Gore PR, Patil CR, Mahajan UB, Sharma C, Talla SP, Ojha SK: Therapeutic Potential and Pharmaceutical Development of Thymoquinone: A Multitargeted Molecule of Natural Origin. Frontiers in pharmacology 2017, 8:656.

27. Achour M, Jacq X, Ronde P, Alhosin M, Charlot C, Chataigneau T, Jeanblanc M, Macaluso M, Giordano A, Hughes AD et al: The interaction of the SRA domain of ICBP90 with a novel domain of DNMT1 is involved in the regulation of VEGF gene expression. Oncogene 2008, 27(15):2187-2197.

28. Keith FJ, Bradbury DA, Zhu YM, Russell NH: Inhibition of bcl-2 with antisense oligonucleotides induces apoptosis and increases the sensitivity of AML blasts to Ara-C. Leukemia 1995, 9(1):131-138.

29. Long J, Fang S, Dai Q, Liu X, Zhu W, Wang S: The Wilms Tumor-1 (WT1) rs16754 polymorphism is a prognostic factor in acute myeloid leukemia (AML): a meta-analysis. Oncotarget 2016, 7(22):32079-32087.

30. Glienke W, Chow KU, Bauer N, Bergmann L: Down-regulation of wt1 expression in leukemia cell lines as part of apoptotic effect in arsenic treatment using two compounds. Leukemia \& lymphoma 2006, 47(8):1629-1638. 